\title{
Very rapid forgetting
}

\author{
PAUL MUTER \\ University of Toronto, Toronto, Ontario MSS IAI, Canada
}

\begin{abstract}
Human forgetting can be much more rapid than previous experiments have indicated. Subjects who do not expect a test after a filled retention interval can rarely recall three consonants correctly after $2 \mathrm{sec}$ of distraction. If there are two kinds of memory, primary and secondary, the present technique provides a purer method of studying forgetting from primary memory.
\end{abstract}

This article calls attention to a remarkable gap in the experimental literature on human memory and describes two attempts to fill this gap.

A large number of texts on human memory contain statements like the following: "If you are ... faced with the situation of having to look up a number in a phone book and then walk to the other side of the room to dial it ... if you don't keep saying it over and over to yourself, it will disappear very quickly" (Cermak, 1972, p. 12). A more general version of this statement would be that if a person encodes a short sequence of items for the purpose of maintaining the sequence in memory for a short period, and he is then distracted, memory for the sequence will be rapidly lost. Despite this widespread interest in the loss of maintained items, to my knowledge there is no good basis for an estimate of how rapidly maintained items are lost under such circumstances, because in light of recent theoretical and experimental developments, largely stemming from Craik and Lockhart (1972), it can be said that no one has adequately studied forgetting under such circumstances.

Similarly, if there are two kinds of memory, primary (active) and secondary (inactive), and if primary memory has a characteristic forgetting rate, there is no good basis for estimating this rate, although many estimates have been offered (e.g., Bjork, 1975, p. 155; Hall, 1971, p. 513; Keele, 1973, p. 20; Miller \& Johnson-Laird, 1976, p. 143).

Many investigators, including Daniels (1895) and Peterson and Peterson (1959) have presented three items to subjects, distracted them for varying periods of time, asked them to recall the three items in order, presented three new items, and so on. Peterson and Peterson found that the probability that all three items will be recalled correctly falls off rapidly as the retention interval

This research was supported by Research Grants APA146 from the National Research Council of Canada and OMHF164 from the Ontario Mental Health Foundation to Bennet $B$. Murdock, Jr. I am grateful for the helpful comments of F. I. M. Craik, J. E. Eich, B. Farell, B. B. Murdock, Jr., D. L. Schacter, E. Tulving, and many other members of the Ebbinghaus Empire, and for H. L. Kaplan's operating system. Requests for reprints may be sent to Paul Muter, Behavioral Research Group, Department of Communications, 300 Slater Street, Ottawa K1A 0C8, Canada. increases, until it is approximately $10 \%$ after $18 \mathrm{sec}$. Such rapid forgetting was regarded as "startling" (Melton, 1963), but this result has been replicated many times.

The Peterson and Peterson (1959) experiment and others like it do not provide adequate information with respect to the rate of loss of maintained items or the rate of loss from primary memory, because in these experiments subjects know during encoding that they will be asked to recall the sequence after a retention interval filled with a distracting task. Ideas of Craik and Lockhart (1972) and data of Jacoby and Bartz (1972) and Watkins and Watkins (1974) suggest that the subject's purpose and expectations during encoding affect the nature of the cognitive processing during encoding and that the nature of the cognitive processing affects the nature and strength of the memory trace and the probability of subsequent retrieval from memory.

Several researchers (e.g., Craik \& Tulving, 1975; Hyde \& Jenkins, 1969) have demonstrated that the probability of successful retrieval from memory depends on the manner in which the subject has been induced, by orienting tasks, to process the inputs (e.g., semantically vs. acoustically). Furthermore, even without an explicit orienting task, it has been demonstrated that subjects perform differently in memory tasks if they expect to be distracted during the retention interval than if they do not, perhaps because they form a secondary memory trace (Jacoby \& Bartz, 1972; Watkins \& Watkins, 1974). Some kinds of cognitive processing are optimal for immediate recall and not optimal for recall after a filled interval, and other kinds of cognitive processing are optimal for recall after a filled interval and not optimal for immediate recall (Mazuryk, 1974; Mazuryk \& Lockhart, 1974).

If a short sequence of items is encoded with the expectation of no distraction before recall, as, for example, when a telephone number is looked up for the purpose of dialing, perhaps processing of the items is different from that in the Peterson and Peterson (1959) experiments, and perhaps forgetting is much more rapid than has been demonstrated to date.

Experiments 1 and 2 were similar in most respects to that of Peterson and Peterson (1959, Experiment 1). The most important difference was that subjects were 
induced to expect rarely or never to be asked to recall after a filled retention interval.

\section{EXPERIMENT 1}

In Experiment 1, each subject experienced a recall test after a filled retention interval only once, near the end of the 20-min experimental session. Most of the remaining trials were set-up trials, in which subjects were required only to maintain items in memory over a 2 -sec unfilled interval.

\section{Method}

Subjects. The 32 subjects were people in the university community who had responded to notices requesting paid subjects for an experiment on information processing. They were not known by the experimenter and were mostly undergraduates. Some had completed psychology courses.

Apparatus and stimuli. Subjects sat in front of a PDP-12A computer, which controlled randomization and presentation of stimuli. The distance between the cathode-ray screen and the subjects' eyes was approximately $75 \mathrm{~cm}$. The height of the uppercase letters forming the stimuli was approximately $8 \mathrm{~mm}$, or $6 \mathrm{deg}$ of visual angle. Subjects' responses were entered via a DECwriter by the experimenter. The trigrams were drawn at random with replacement from the pool of all possible sequences of three different consonants, excluding $Y$.

Procedure. A random $78 \%$ of the 128 experimental trials were maintenance trials (see Figure 1). On maintenance trials, a trigram was presented on the screen for $1 \mathrm{sec}$. Two seconds later, the message "LETTERS?" appeared on the screen. Subjects had been instructed beforehand to recall aloud, in order, the last three letters they had seen whenever the message "LETTERS?" appeared. Subjects had as much time as they wanted to recall the trigram. (In Figure 1, self-pacing is indicated by a broken horizontal line.)

A random $17 \%$ of the experimental trials were counting trials (see Figure 1). On counting trials, a trigram appeared on the screen for $1 \mathrm{sec}$, followed immediately by a random three-digit number, from which the subjects had been instructed to count backwards by threes as quickly and accurately as possible. Six seconds after the onset of the digits, the next trial began. The purpose of the counting trials was to familiarize subjects with the task of counting backward. During the 22 practice trials, subjects were reminded to perform as quickly and accurately as

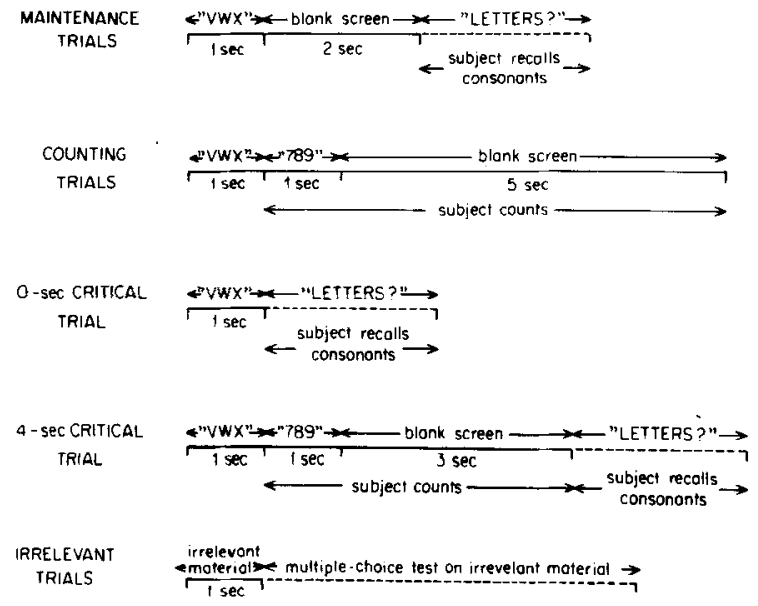

Figure 1. Procedures in Experiment 1. possible on the distractor task, especially at the beginning of the distractor period.

A random 3\% of the experimental trials were irrelevant trials (see Figure 1), included to disguise the purpose of the experiment. These very difficult trials consisted of either a sentence followed by a multiple-choice comprehension test or a string of numbers to be added followed by a multiple-choice test in which different sums were the alternatives. In each case, the input was presented for only $1 \mathrm{sec}$. The last trial of the session was an irrelevant trial.

There were only two critical trials per session in Experiment 1 (see Figure 1). The 4-sec critical trial was essentially a Peterson and Peterson (1959) trial: A trigram appeared on the screen for $1 \mathrm{sec}$, followed immediately by a three-digit number, from which the subject counted backward by threes, followed by the message "LETTERS?" $4 \mathrm{sec}$ after the onset of the number. On the 0 -sec critical trial, the message "LETTERS?" appeared immediately (25 msec) after the offset of the letters. For half the subjects, the $4-\mathrm{sec}$ critical trial appeared on the 98th experimental trial and the 0 sec critical trial appeared on the 118th experimental trial, and for half this order was reversed.

Before the session, the subjects read the following instructions.

"Please keep your eyes on the screen at all times.

"Read (silently) whatever appears on the screen.

"When the message "LETTERS" appears on the screen, say aloud, in order, the last three letters you have seen.

"When a three-digit number appears on the screen, say the number aloud, and count backwards by threes as quickly and accurately as you can. For example, if the number is "391," you should say "three ninety-one, three eighty-eight . . .," etc., as quickly and accurately as possible until something else appears on the screen. (Your voice will be recorded so that the speed and accuracy of your counting backwards can be determined.)

"Do not talk to the experimenter after the practice trials.

"If ever you don't know the answer, just guess. Some of the tasks are very difficult, so do not expect to get everything correct."

Thus, although subjects were not told that they would not be tested for recall after a filled interval, they were not told that they would be.

After the subject read the instructions and before the practice trials began, the experimenter asked the subject questions about critical aspects of the procedure and did not proceed until the subject had stated that when the message "LETTERS?" appeared on the screen, he was to recall aloud, in order, the last three letters he had seen. The 22 practice trials consisted of 10 maintenance trials, 10 counting trials, and 2 irrelevant trials.

At the end of the session, the subject was not informed of the purpose of the experiment and was asked not to discuss the experiment with any potential future subjects.

\section{Results and Discussion}

Three subjects were replaced: two who were not counting rapidly enough and one who recalled the digits on the critical trial before attempting to recall the consonants. They correctly recalled two, zero, and zero consonants, respectively, on the 4-sec critical trial.

Forgetting in Experiment 1 was several times more rapid than in earlier experiments using the Peterson and Peterson (1959) procedure. The proportion of trigrams recalled correctly is plotted in Figure 2. For purposes of comparison, analogous data from a typical experiment (Murdock, 1961, Experiment 1) using the Peterson and Peterson procedure are also plotted.

A more sensitive measure is the proportion of individual consonants recalled correctly without regard to order of recall. This result is plotted in Figure 3. The 


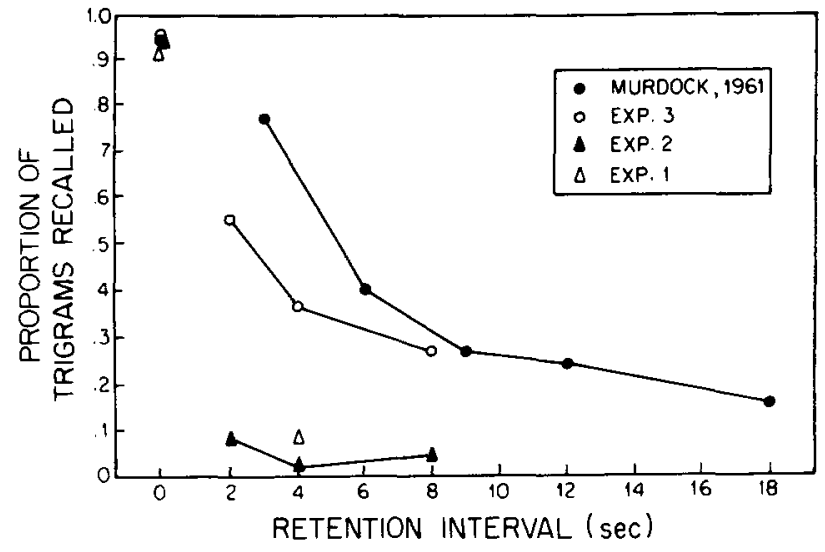

Figure 2. Proportion of trigrams correct on critical trials as a function of retention interval.

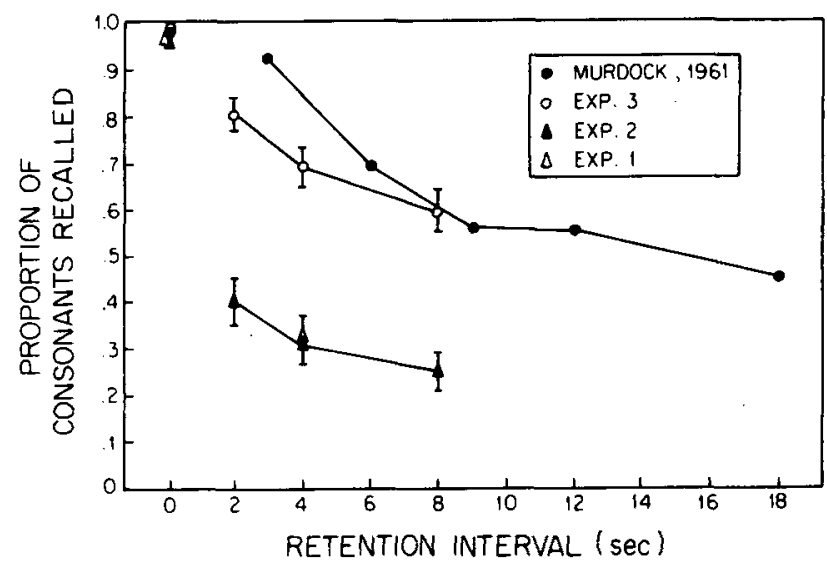

Figure 3. Proportion of individual consonants correct on critical trials without regard to order of recall as a function of retention interval. Chance performance is approximately $15 \%$ in Experiment 1 and 19\% in Experiments 2 and 3. The bars indicate standard errors.

difference between Experiment 1 results and Murdock's (1961) results is actually greater than shown in Figure 2, since in Murdock's experiment, subjects were not required to guess and did not respond for $12 \%$ of the consonants.

On maintenance trials, the proportion of trigrams recalled correctly was 97 .

The objection could be raised that the element of surprise on the 4-sec critical trial produced the poor performance. It is possible that for some subjects the functional retention interval was 5 or $6 \mathrm{sec}$ on the 4-sec critical trial because of a surprise element, but casual observation revealed that most subjects shrugged, lifted their hands, or gave some other sign of frustration within approximately $1 \mathrm{sec}$ after the message "LETTERS?" appeared on the 4-sec critical trial. Subjects appeared to know immediately what was required, perhaps because during the instruction period pains were taken to elicit from subjects a statement of what they were required to do when the message "LETTERS?" appeared. In any event, the element of surprise played little or no role in Experiment 2, in which subjects were told beforehand that occasionally they would be tested after a filled retention interval.

\section{EXPERIMENT 2}

Experiment 2 was designed to replicate Experiment 1 under somewhat different conditions. The major difference was that subjects were told beforehand that on $2 \%$ of the trials they would be asked to recall after a filled retention interval. It was hoped that such a low percentage would induce maintenance processing. This procedure has three advantages over the procedure of Experiment 1. First, it is more efficient. More critical trials per subjecthour can be run. Second, it reduces the importance of the intersubject communication problem; in Experiment 1 , any intersubject communication with respect to the 4-sec critical trial may have altered performance on this trial. Third, it reduces the element of surprise.

\section{Method}

Subjects. The 24 subjects were recruited in the same way from the same population as in Experiment 1. No subject participated in both experiments.

Apparatus and Stimuli. The apparatus and stimulus were the same as in Experiment 1, except that trigrams including the highfrequency consonants $\mathrm{N}, \mathrm{R}, \mathrm{S}$, and $\mathrm{T}$ were dropped from the trigram pool.

Procedure. The procedure was the same as for Experiment 1, with the following exceptions.

Instead of counting trials, Experiment 2 included reading trials. On reading trials, a trigram appeared for $1 \mathrm{sec}$, followed by 36 words presented 4 at a time at a $1-\mathrm{sec}$ rate for a total of $9 \mathrm{sec}$. (The words were drawn randomly on each trial from the Toronto word pool, a pool of 1,080 common English twosyllable words not more than eight letters long, with homophones, contractions, archaic words, and proper nouns excluded.) Subjects read aloud as many of the words as possibie. Then a three-alternative forced-choice recognition test of the words just presented appeared on the screen. On these word-recognition tests, subjects received immediate feedback.

On maintenance trials, the retention interval was $1.5 \mathrm{sec}$ instead of 2 sec.

On critical trials in Experiment 2, the distracting task was reading, not counting, but there was no word-recognition task. In the 45 -min session, there were eight critical experimental trials, two each at retention intervals of $0,2,4$, and $8 \mathrm{sec}$. Each subject received the four critical trials in a different order, repeated twice. The eight critical trials were scattered at random among the 340 experimental trials, with the constraint that at least 14 trials intervened between successive critical trials. In addition, one $0-\mathrm{sec}$ critical trial and one $4 \mathrm{sec}$ critical trial were included in the 22 practice trials. Critical trials were always preceded by maintenance trials.

of the 340 experimental trials, the percentages of critical, maintenance, reading, and irrelevant trials were $2 \%, 84 \%, 9 \%$, and $5 \%$, respectively. Of the 22 practice trials, 2 were critical trials, 10 were maintenance trials, 8 were reading trials, and 2 were irrelevant trials.

To further decrease the probability that subjects would encode the consonants in a way suitable for recall after a filled interval and to further decrease the probability that subjects would rehearse the consonants during the distractor period, sub- 
jects were told before the session began not to be too concerned if they could not recall the consonants after the filled interval.

In Experiment 2, the very difficult irrelevant trials had the additional purpose of enhancing subjects' tolerance for failure.

Following are the complete instructions for Experiment 2.

"Please keep your eyes on the screen at all times; a variety of things will appear there.

"On most trials, you will see three letters. Later, when the message "LETTERS?" appears on the screen, say the three letters aloud, in order.

"Whenever you see four words, one above the other, read aloud the words as quickly as you can, and try to remember them. Sometimes your memory for them will be tested, and you will receive an extra three cents for each time you are correct (for a maximum possible bonus of 96 cents).

"On less than two percent of the trials, you will see three letters, then you will read some words, then you will be asked to recall the letters. Do not be too concerned if you cannot recall the letters at this point; just guess if necessary.

"Do not talk to the experimenter after the practice trials.

"Some of the tasks are very difficult, so do not expect to get everything correct. Guess if necessary."

\section{Results and Discussion}

The proportion of trigrams recalled correctly and the proportion of consonants recalled correctly are plotted in Figures 2 and 3, respectively. In Figure 3, correct guessing inflates the results for Experiment 2 even more than for Experiment 1, since in Experiment 2 the number of consonants used was 16 instead of 20 . On maintenance trials, the proportion of trigrams recalled correctly was .94 .

These results include one subject who was several standard deviations better than the second best subject on critical trials: He recalled virtually everything. Later he revealed that he was attempting to generate semantic associations for all trigrams.

The results for Experiment 2 were quite similar to the results for Experiment 1, despite the difference in distracting tasks. This finding is consistent with the conclusion of Craik and Levy (1976), who reviewed the literature on forgetting from primary memory and reported that the exact nature of the distracting task has little or no effect, provided that both the to-be-remembered items and the distracting task are verbal.

\section{EXPERIMENT 3}

Experiments 1 and 2 differed from the classic Peterson and Peterson (1959) experiments in several minor details in addition to the difference in the probability of a recall test after a filled interval. (The purpose of Experiments 1 and 2 was to attempt to provide the best possible answers to the two questions raised in the introduction; the purpose was not to replicate the method of Peterson and Peterson in all respects but one.) Experiment 3 was designed to provide some information about the transition between the traditional experiments and Experiments 1 and 2 .

In most respects Experiment 3 was identical to Experiment 1. The key difference was in the proportions of the various types of trials: In Experiment 3, on the vast majority of trials, subjects were asked to recall the trigram after a filled interval.

\section{Method}

Subjects. The 16 subjects were recruited in the same way from the same population as in Experiments 1 and 2, except that Experiment 3 was run 1 year later.

Apparatus and Stimuli. The apparatus and stimuli were the same as in Experiment 1 , except that $N, R, S$, and $T$ were excluded, as in Experiment 2.

Procedure. The procedure was identical to that for Experiment 1, with two exceptions: The proportions of the various types of trials were different, and the retention interval on critical trials was $0,2,4$, or $8 \mathrm{sec}$.

Of the 104 experimental trials in the 20-min session, 92\% were critical trials, $2 \%$ were maintenance trials, $2 \%$ were counting trials, and $4 \%$ were irrelevant trials. Of the 96 critical experimental trials, there were $6,24,30$, and 36 at retention intervals of $0,2,4$, and $8 \mathrm{sec}$, respectively. There were 12 practice trials: 8 critical trials, 1 maintenance trial, 1 counting trial, and 2 irrelevant trials. In addition, just before the experimental trials, there were four critical trials that were not scored.

The instructions were identical to those in Experiment 1.

\section{Results and Discussion}

One subject, who did not count rapidly enough, was replaced.

The proportion of trigrams recalled correctly and the proportion of consonants recalled correctly are plotted in Figures 2 and 3, respectively. In Experiment 3, performance at 2 and $4 \mathrm{sec}$ was somewhat worse than would be expected on the basis of Murdock's (1961) data. This suggests that a small proportion of the difference between Experiment 1 and 2 results and earlier results is due to differences other than the difference in the probability of a recall test after a filled interval. Perhaps subjects in the late 1970s are inferior to subjects in the late 1950s and early 1960s. Perhaps subjects "cheated" less (rehearsed the trigrams less) during the distractor task in the present experiments than in the earlier ones: In Experiments 1 and 3, subjects could see the tape recorder in operation and were told that the speed and accuracy of their counting backward would be computed; in Experiment 2, subjects were given bonus money for good performance on the distractor task.

Experiment 3 data are, however, much more similar to Murdock's (1961) data than to the data of Experiments 1 and 2 . Thus, although some ignorance remains about the transition between the traditional experiments and Experiments 1 and 2, the Experiment 3 results strongly suggest that the rapid forgetting in Experiments 1 and 2 was largely due to the low probability of a recall test after a filled interval.

\section{GENERAL DISCUSSION}

To my knowledge, Experiments 1 and 2 produced the most dramatic human forgetting ever demonstrated, not including sensory memory studies or clinical studies.

There are several possible explanations of the difference in forgetting rates between Experiments 1 and 2, 
on the one hand, and the classic Peterson and Peterson (1959) experiments and Experiment 3, on the other hand. It is possible that the difference is due to less cheating during the distractor period. Another possibility is that "directed forgetting" is responsible for the difference: Perhaps there is no difference at encoding time and no difference in the amount of cheating, but solely a difference in the extent to which subjects "actively erase or dump" items from memory (Bjork, 1970 , p. 263). A third interpretation is that the difference is due to the nature of the cognitive processing while the consonants were on the screen. The subjects may have processed the consonants in a way that is effective for maintenance but ineffective for recall after a filled interval. For example, perhaps the subjects were less likely to form a secondary memory trace in Experiments 1 and 2 than in the other experiments.

It is unlikely that Experiments 1 and 2 reflect loss from sensory memory only. Haber and Standing (1970) have provided convincing evidence that the duration of visual persistence decreases as stimulus duration increases, such that visual persistence plays a negligible role if stimulus duration is above 250 msec. Even if this conclusion is wrong and there is visual persistence when presentation time is a full $1 \mathrm{sec}$, on maintenance trials the retention interval was longer than most estimates of the duration of visual persistence, and on these trials the trigrams were recalled correctly on $97 \%$ of the trials in Experiment 1 and 94\% of the trials in Experiment 2. Furthermore, subjects would have difficulty relying on sensory memory for near-perfect performance on the 0 -sec critical trials, because the message "LETTERS?" appeared at the same location on the screen as the trigram.

The present experiments are important for at least four partially overlapping reasons. First, for those who favor a distinction between primary memory and second. ary memory, Experiments 1 and 2 provide a new method of studying forgetting from primary memory that is less contaminated by secondary memory involvement than were earlier methods. Much of the theorizing in the area of primary memory in the past 2 decades has been based on results from the Peterson and Peterson (1959) procedure and other procedures (e.g., Shiffrin \& Cook, 1978 ) in which subjects knew during encoding that they would be asked to recall items after a period of distraction, and therefore in which the formation of a secondary memory trace was likely (Jacoby \& Bartz, 1972; Watkins \& Watkins, 1974). Perhaps some theoretical issues should be reopened under conditions like those in Experiments 1 and 2. Under such conditions, for example, the nature of the distractor (e.g., verbal vs. nonverbal) may be irrelevant to the rate of forgetting, and there may be no proactive interference or intraunit interference. (Whether prior trigrams had an interfering effect in Experiments 1 and 2 is unknown.) The absence of such interference effects, which are pervasive in long-term tasks, would reinforce the distinction between primary memory and secondary memory. In addition, Experiments 1 and 2 provide a better estimate of the approximate rate of forgetting from primary memory. There is probably no fixed rate of forgetting from primary memory, but it may always be of a certain order of magnitude, and the present experiments suggest that it is not the order of magnitude previously thought. (A memory buffer with such a rapid forgetting rate might appear to be relatively unuseful, but if there is no distraction, items in primary memory may be refreshed periodically, and therefore a certain number of items may be maintained indefinitely.)

Forgetting rates in Experiments 1 and 2 were substantially more rapid than that computed for primary memory by Waugh and Norman (1965, Figure 9). Waugh and Norman assumed that the amount of secondary memory involvement is indicated by the asymptote, and they subtracted this component from the Murdock (1961) data to produce an estimate of forgetting from primary memory. A possible means of resolving this discrepancy is to reject the assumption that the amount of secondary memory involvement is indicated by the asymptote alone. In the Murdock data, the rate may reflect some forgetting from secondary memory in addition to forgetting from primary memory. I know of no compelling reason to cling to the assumption that the asymptote reflects secondary memory and the rate reflects primary memory, and, as Crowder (1976, p. 215) has observed, the experimental evidence does not convincingly demonstrate that certain variables affect only the rate and certain other variables affect only the asymptote. If the rate reflects some forgetting from secondary memory, then the Waugh and Norman subtraction method yields an underestimate of the rate of forgetting from primary memory.

Second, Experiments 1 and 2 represent an improved means of studying what happens if a short sequence of items is input for maintenance only (as when a telephone number is looked up for the purpose of dialing) and a distraction occurs. As stated in the introduction, this question has drawn the attention of many psychologists, but before the 1970s, prevailing assumptions did not encourage a suitable attempt to answer it.

Third, these data provide a new answer to the basic empirical question: How rapid can forgetting be? It can be at least as rapid as demonstrated in Figures 2 and 3 , several times more rapid than previously thought. (Such rapid forgetting may enable subjects to optimize performance across tasks under the present circumstances. For a discussion of the adaptiveness of the skill of rapid forgetting, see Bjork, 1970). Of course, it is possible that even more dramatic forgetting could be produced: In Experiment 2, one subject apparently did prepare for recall following a filled interval, and others may have done this occasionally. In addition, some trigrams may have been meaningful to particular subjects. Beyond restricting the vocabulary of letters used, no attempt was made to weed out meaningful trigrams. 
Fourth, the present results lend support to the proposition that subjects' expectations with respect to the retention interval and subjects' purposes play a crucial role in memory phenomena. The essential difference between the classic Peterson and Peterson (1959) experiments and Experiment 3 on the one hand, and Experiments 1 and 2 on the other hand, was that, in the latter, only maintenance was required on most trials; a test after a filled retention interval was not normally expected. This difference produced dramatically worse memory performance.

\section{REFERENCES}

BJork, R. A. Positive forgetting: The noninterference of items intentionally forgotten. Journal of Verbal Learning and Verbal Behavior, 1970, 9, 255-268.

Bјonk, R. A. Short-term storage: The ordered output of a central processor. In F. Restle, R. M. Shiffrin, N. J. Castellan, H. R. Lindman, \& D. B. Pisoni (Eds.), Cognitive theory (Vol. 1). Hillsdale, N.J: Erlbaum, 1975.

CеRмaK, L. S. Human memory: Research and theory. New York: Ronald Press, 1972.

Craik, F. I. M., \& Levy, B. A. The concept of primary memory. In W. K. Estes (Ed.), Handbook of learning and cognitive processes (Vol. 4). Hillsdale, N.J: Erlbaum, 1976.

Craik, F. I. M., \& Lockhart, R. S. Levels of processing; A framework for memory research. Journal of Verbal Learning and Verbal Behavior, 1972, 11, 671-684.

Craik, F. I. M., \& Tulving, E. Depth of processing and the retention of words in episodic memory. Journal of Experimental Psychology: General, 1975, 104, 268-294.

Crowder, R. G. Principles of learning and memory. Hillsdale, N.J: Erlbaum, 1976.

Daniels, A. H. The memory after-image and attention. American Journal of Psychology, 1895, 6, 558.564.

Haber, R. N., \& Standing, L. Direct estimates of apparent duration of a flash followed by visual noise. Canadian Journal of Psychology, 1970, 24, 216-229.

HaLL, J. F. Verbal leurning and retention. New York: Lippincott, 1971.

Hyde, T. S., \& JENKins, J. J. The differential effects of incidental tasks on the organization of recall of a list of highly associated words. Journal of Experimental Psychology, 1969, $82,472-481$.

$\mathrm{J}_{\mathrm{ACOBY}}$, L. L., \& BARTZ, W. H. Encoding processes and the negative recency effect. Journal of Verbal Learning and Verbal Behavior, 1972, 11, 561-565.

KeEle, S. W. Attention and human performance. Pacific Palisades, Calif: Goodyear, 1973.

MAzUryk, G. F. Positive recency in final free recall. Journal of Experimental Psychology, 1974, 103, 812-814.

MazUryк, G. F., \& LockнarT, R. S. Negative recency and levels of processing in free recall. Canadian Journal of Psychology, 1974, 28, 114-123.

MELton, A. W. Implications of short-term memory for a general theory of memory. Journal of Verbal Learning and Verbal Behavior, 1963, 2, 1-21.

Miller, G. A., \& Johnson-Laird, P. N. Language and perception. Cambridge: Cambridge University Press, 1976.

Murvock, B. B., JR. The retention of individual items. Journal of Experimental Psychology, 1961, 62, 618-625.

Peterson, L. R., \& Peterson, M. J. Short-term retention of individual verbal items. Journal of Experimental Psychology, 1959, 58, 193-198.

Shifrrin, R. M., \& Cook, J. R. Short-term forgetting of item and order information. Journal of Verbal Learning and Verbal Behavior, 1978, 17, 189-218.

Watkins, M. J., \& Watkins, O. C. Processing of recency items for free recall. Journal of Experimental Psychology, 1974, $102,488-493$

Wavgh, N. C., \& Norman, D. A. Primary memory. Psychological Review, 1965, 72, 89-104.

(Received for publication August 3, 1979; revision accepted November $26,1979$. .) 